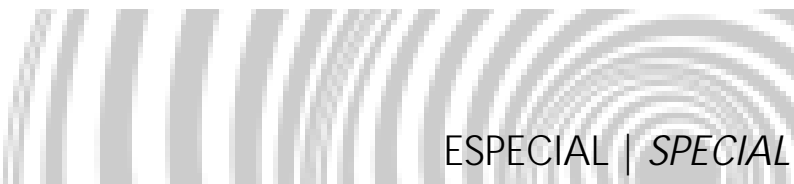

\section{Causas y consecuencias de la deficiencia de hierro}

\section{Causes and consequences of iron deficiency}

Manuel OLIVARES ${ }^{1}$

Tomás WALTER ${ }^{1}$

La deficiencia de hierro es la deficiencia nutricional más prevalente y la principal causa de anemia a escala mundial. Además de las manifestaciones propias de la anemia, se han descrito otras manifestaciones no hematológicas tales como: disminución de la capacidad de trabajo físico y de la actividad motora espontánea, alteraciones de la inmunidad celular y de la capacidad bactericida de los neutrófilos, disminución de la termogénesis, alteraciones funcionales e histológicas del tubo digestivo, falla en la movilización de la vitamina A hepática, mayor riesgo de parto prematuro, bajo peso de nacimiento y de morbilidad perinatal, menor transferencia de hierro al feto, una disminución de la velocidad de crecimiento, alteraciones conductuales y del desarrollo mental y motor, velocidad de conducción más lenta de los sistemas sensoriales auditivo y visual, y reducción del tono vagal. La prevención de la deficiencia de hierro incluye cambios en los hábitos alimentarios, fortificación de los alimentos y la suplementación con hierro.

Términos claves: deficiencia de hierro, anemia, lactantes, niño, embarazo, desarollo mental.

\section{A B S T R A C T}

Iron deficiency is the most prevalent nutritional deficiency and the main cause of anemia worldwide. Apart from anemia effects, other non-hematological manifestations have been described, such as decreased capacity for physical work and spontaneous motor activity, impairment of cell-mediated immunity and bactericidal capacity of neutrophils, decreased thermoregulation, functional and histological abnormalities of the gastrointestinal tract, defective mobilization of liver vitamin A, increased risk of premature labor, low birthweight and perinatal morbidity, reduced iron transfer to the fetus and growth retardation, behavioral disorders and mental and motor development delays, decreased auditory and visual systems nerve conduction velocity, as well as reduction of the vagal tone. The prevention of iron deficiency can be accomplished by changes in dietary patterns, food fortification and iron supplementation.

Index terms: iron deficiency, anemia, infants, child, pregnancy, mental development. 
6 M. OLIVARES \& T. WALTER

\section{INTRODUCCIÓ N}

El hierro pese a encontrarse en cantidades muy pequeñas en nuestro organismo, participa como cofactor en numerosos procesos biológicos indispensables para la vida, tales como el transporte de oxígeno, fosforilación oxidativa, metabolismo de neurotransmisores y la síntesis de ácido desoxiribonucleico ${ }^{1}$.

La deficiencia de hierro es la deficiencia nutricional más prevalente a escala mundial y la principal causa de anemia². En los países en vías de desarrollo los grupos mas afectados son los niños debido a los mayores requerimientos determinados por el crecimiento, y en la mujer en edad fértil por la pérdida de hierro debida al sangramiento menstrual o a las mayores necesidades de este mineral requeridas por el embarazo. Este aumento de las necesidades no es cubierto por la dieta habitual la que tiene cantidades insuficientes de hierro y/o presenta una baja biodisponibilidad de este nutriente (predominante en inhibidores de la absorción de hierro y con un bajo contenido de hierro hemínico) $)^{3}$.

En la infancia la causa más frecuente de la carencia de hierro es la nutricional, originada por la dificultad de cubrir los mayores requerimientos de este mineral por la dieta habitual, predominantemente láctea ${ }^{4}$. Esta predisposición a desarrollar anemia es aún mayor en el prematuro debido a sus menores depósitos de hierro al nacer y requerimientos de hierro más elevados. Esta susceptibilidad también es mayor en el niño con lactancia artificial, a menos que reciba fórmulas lácteas fortificadas, ya que el contenido de hierro de la leche de vaca es bajo y este mineral es pobremente absorbido, solo un $4 \%{ }^{5}$. Por el contrario el lactante de término alimentado con leche materna exclusiva, pese al bajo contenido de hierro de ésta, se encuentra protegido hasta los 6 meses de vida debido a la excelente biodisponibilidad del hierro de esta leche, un $50 \%$.
En el niño mayor debido a la disminución del ritmo de crecimiento y a una dieta más abundante y variada, la etiología nutricional es menos prevalente, siendo habitualmente a esta edad la deficiencia una situación que se arrastra desde el período de lactante ${ }^{4}$. En esta etapa de la vida adquieren importancia otras causas, especialmente las pérdidas sanguíneas aumentadas y el síndrome de malabsorción. De los sangramientos el más frecuente es el digestivo. En las regiones tropicales una causa común de pérdida crónica de sangre son infestaciones por parásitos intestinales hematófagos, como la ancilostomiasis y la trichocefalosis masiva. La malaria, en regiones donde es endémica aumenta la incidencia de la anemia, pero no se debe exclusivamente al déficit de hierro.

En la mujer en edad reproductiva la pérdida de hierro por la menstruación determina un aumento de los requerimientos de este mineral, lo que hace que este grupo sea más vulnerable a experimentar una deficiencia de hierro ${ }^{7}$. Existen importantes variaciones individuales en la pérdida de hierro por la menstruación, sin embargo en una misma mujer esta variación entre diferentes períodos es pequeña. Por otra parte los métodos anticonceptivos pueden alterar significativamente la pérdida menstrual. La pérdida de hierro es menor en mujeres que utilizan anticonceptivos orales y mayor que lo normal cuando utilizan dispositivos intrauterinos ${ }^{8}$. El requerimiento de hierro para afrontar un embarazo es de $1040 \mathrm{mg}$. De esta cifra $350 \mathrm{mg}$ son entregados al feto $y$ placenta y $250 \mathrm{mg}$ se pierden con el sangramiento del parto. En el perio do de gestación se necesitan $450 \mathrm{mg}$ para cubrir la demanda impuesta por la expansión de la masa eritrocitaria materna. Las pérdidas basales normales continúan, excepto la pérdida menstrual, lo que suma aproximadamente $240 \mathrm{mg}$. Sin embargo, la pérdida neta de hierro es de $840 \mathrm{mg}$ (1040mg - $250 \mathrm{mg}$ perdidos en el parto $+450 \mathrm{mg}$ recuperados en el post parto al contraerse la masa eritrocitaria) ${ }^{9}$. Esta pérdida neta es mayor en partos por cesárea, ya que el sangramiento es casi el doble que en un parto normal. 
Los requerimientos de hierro son desiguales durante el embarazo. La cantidad promedio de hierro absorbido requerido diariamente es de $0,8 \mathrm{mg}$ en el primer trimestre (incluso menor que el la mujer no gestante), concentrándose la mayor parte de los requerimientos en los dos últimos trimestres, 4,4mg en el segundo trimestre y $6,3 \mathrm{mg}$ en el tercer trimestre en mujeres que comienzan su embarazo con depósitos ausentes o mínimos ${ }^{10}$. Por otra parte, la absorción de hierro dietario es baja en el primer trimestre, para luego aumentar progresivamente a medida avanza el embarazo, llegando a triplicarse alrededor de la semana 36 de gestación ${ }^{9}$. No obstante este aumento, es imposible cubrir los elevados requerimientos sólo con el aporte de hierro de la dieta. Se estima, que a pesar del aumento de la absorción de hierro, se requieren entre 300 a $500 \mathrm{mg}$ de depósitos de hierro previo a la concepción para cubrir el déficit neto de hierro impuesto por el embarazo ${ }^{9}$. Esta cuantía de depósitos de hierro es difícil de encontrar aún en sociedades con altos ingresos económicos. En un grupo de 343 adolescentes embarazadas del área sur oriente de Santiago, un 77\% tenía depósitos de hierro inferiores a $300 \mathrm{mg}$ durante el primer trimestre de gestación y un $95 \%$ inferiores a $500 \mathrm{mg}^{11}$. En embarazadas multíparas, con períodos intergestacionales cortos, esta condición suele ser aún peor. En un estudio realizado en Santiago, un 27\% de mujeres adultas tenía depósitos de hierro depletados en el primer trimestre de la gestación ${ }^{12}$.

Cuando el aporte de hierro es insuficiente para cubrir los requerimientos se producen etapas progresivas de severidad de la deficiencia de hierro ${ }^{4,13}$. Primero se agotan los depósitos (deficiencia latente) que se caracteriza por una disminución de la Ferritina Sérica (FS). Si el aporte insuficiente continúa se compromete el aporte de hierro tisular (eritropoyesis deficiente en hierro) que se caracteriza precozmente por un aumento de los Receptores de Transferrina Séricos (RTf) y más tarde por una disminución de la Saturación de la Transferrina (Sat) y aumento de la Protoporfirina Libre Eritrocitaria (PLE). Finalmente al persistir el balance negativo se llega a la etapa más severa, caracterizada por una anemia microcítica hipocroma.

La forma ideal de prevenir la carencia de hierro es mediante una dieta adecuada, lo que no siempre es posible de lograr por limitaciones económicas o hábitos muy arraigados. La fortificación de los alimentos con hierro es la forma más práctica de prevenir la carencia de hierro ${ }^{14}$. Su principal ventaja es que el consumo de estos productos no requiere de una conducta activa del sujeto. Cuando no se está consumiendo alimentos fortificados con hierro o se requiere proveer una gran cantidad de hierro en un período corto (embarazo o anemia clínica), se recomienda la suplementación con hierro medicinal ${ }^{14}$. La efectividad de esta medida se ve principalmente limitada por la dificultad de mantener la motivación de consumir el medicamento en individuos sanos, así como el hecho que algunos individuos pueden experimentar molestias gastrointestinales, especialmente cuando se utilizan dosis altas. La incidencia de estos efectos adversos se puede disminuir cuando se utilizan compuestos de hierro protegidos, los que liberan mas gradualmente el hierro iónico a nivel intestinal, o se recurre a una suplementación intermitente en la cual el hierro es administrado una vez a la semana.

En Chile, la anemia ferropriva no es un problema de consideración en preescolares, escolares y hombre adulto y que la prevalencia en el lactante ha disminuido notablemente a cerca de un $8 \%$. En la mujer en edad fértil la prevalencia es de $8 \%$ a $10 \%$ y aumenta a un $20 \%$ en embarazadas durante el segundo y tercer trimestre de gestación. Este perfil de prevalencia de anemia se explica por los programas nacionales de fortificación de alimentos. En Chile desde la década del 50 la harina de trigo está fortificada por ley con hierro, como sulfato ferroso, $30 \mathrm{mg} / \mathrm{kg}$ y a partir de 1999 la leche que entrega el Programa Nacional de Alimentación Complementaria fue fortificada con $10 \mathrm{mg}$ de hierro, como sulfato ferroso, $5 \mathrm{mg}$ de cinc, $0,5 \mathrm{mg}$ de cobre y $70 \mathrm{mg}$ de 
8 M. OLIVARES \& T. WALTER

ácido ascórbico por $100 \mathrm{~g}$ de polvo. En el embarazo, dado los altos requerimientos en un corto período de tiempo, la estrategia a utilizar es la suplementación preventiva, la que se indica a partir del segundo semestre de la gestación.

\section{Consecuencias de la deficiencia de hierro}

Las manifestaciones de la carencia de hierro derivan de aquellas propia de la anemia, y de otras no hematológicas causadas por una malfunción de las enzimas hierro dependientes. Se han descrito alteraciones de la capacidad de trabajo físico y de la actividad motora espontánea, alteraciones de la inmunidad celular y de la capacidad bactericida de los neutrófilos, una controvertida mayor susceptibilidad a las infecciones especialmente del tracto respiratorio, disminución de la termogénesis, alteraciones funcionales e histológicas del tubo digestivo, falla en la movilización de la vitamina A hepática, mayor riesgo de parto prematuro y de morbilidad perinatal, menor transferencia de hierro al feto, disminución de la velocidad de crecimiento, alteraciones conductuales y del desarrollo mental y motor, velocidad de conducción más lenta de los sistemas sensoriales auditivo y visual, y reducción del tono vagal16-22.

Analizaremos en mas detalles los efectos de la deficiencia de hierro sobre la esfera cognitiva y conductual, así como sobre el embarazo y su producto.

\section{Consecuencias de la deficiencia de hierro en el embarazo}

Desde hace ya bastante tiempo se ha estudiado la relación entre los niveles maternos de hemoglobina o hematocrito y el curso del embarazo. Existe una asociación entre el hematocrito/hemoglobina materna, parto prematuro (<37 semanas de gestación), bajo peso de nacimiento $(<2.500 \mathrm{~g})$ y morbi-mortalidad perinatal23-30. Hay evidencias que la asociación entre hemoglobina baja y parto prematuro se da sólo en los dos primeros trimestres degestación ${ }^{24,27-30}$. Por otra parte existe una relación entre la severidad de la anemia y el curso del embarazo. Zhou et al. ${ }^{28}$, en 829 embarazadas, demostraron una asociación entre la severidad de la anemia en el primer trimestre y el riesgo de bajo peso de nacimiento y parto prematuro. El riesgo de parto prematuro aumentó en 1,6 veces con hemoglobina entre $10 \mathrm{~g} / \mathrm{dL}$ y $10,9 \mathrm{~g} / \mathrm{dL}$, en 2,6 veces con hemoglobina entre $9 \mathrm{~g} / \mathrm{dL}$ y $9,9 \mathrm{~g} / \mathrm{dL}$ y en 3,7 veces con hemoglobina entre $6 \mathrm{~g} / \mathrm{dL}$ y $8,9 \mathrm{~g} /$ dl. Por otro lado se ha observado un aumento de parto prematuro, bajo peso de nacimiento y muerte fetal con valores altos de hematocrito/ hemoglobina ( $>13 \mathrm{~g} / \mathrm{dL})^{23,24,31,32}$. En las relaciones en forma de " $U$ " habitualmente los factores que actúan en los extremos son diferentes. En el extremo inferior es probablemente la deficiencia de hierro, mientras en el nivel alto son los procesos hipertensivos del embarazo, particularmente la preeclampsia, en que el aumento de la concentración de hemoglobina se acompañan de una reducción del volumen plasmático con el consiguiente aumento relativo de la concentración de la hemoglobina, aumento de la viscosidad sanguínea, disminución de la perfusión tisular, placentaria y fetal, y alteraciones de la microcirculación con un aumento del riesgo de tromboembolismo ${ }^{31}$.

Si bien la deficiencia de hierro es la principal etiología de la anemia de embarazo, ésta también puede deberse a otras condiciones tales como otras deficiencias nutricionales (folato, vitamina A), infección/inflamación y hemodilución ${ }^{33}$.

Los estudios en los que se ha evaluado el efecto de la anemia ferropriva sobre el embarazo han demostrado el efecto que la anemia ferropriva que ocurre tempranamente en el embarazo se asocia a un riesgo relativo 2,66 veces mayor de parto prematuro y 3,1 veces de bajo peso de nacimiento ${ }^{30}$. El riesgo de parto prematuro 5 veces mayor cuando se le añadía una metrorragia previa 0 concurrente ${ }^{30}$. Al seguir controlando a estas 
embarazadas no se observa un mayor aumento del riesgo después de la semana 38.

La suplementación con hierro de la embarazada, en poblaciones con una alta prevalencia de anemia ferropriva, especialmente al comenzar precozmente, no produce un mayor riesgo de valores elevados de hemoglobina. Por el contrario mejora su nutrición de hierro, aumenta la duración de la gestación y el peso de nacimiento ${ }^{33}$.

Hasta hace no mucho tiempo se pensaba que la nutrición de hierro de la madre no tenía ningún impacto sobre la nutrición de hierro del recién nacido y lactante, salvo en casos de una deficiencia materna de hierro severa ${ }^{33}$. Esta falta de relación obedecía mas bien a problemas metodológicos. Estudios realizados en países en los que la deficiencia de hierro en la embarazada es alta, han mostrado una asociación entre la nutrición de hierro materna y los niveles de ferritina sérica en el cordón ${ }^{34-37}$. Los niveles de ferritina de lactantes menores hijos de madres que recibieron suplementación con hierro durante el embarazo son significativamente más elevados que los de hijos de madres no suplementadas con hierro ${ }^{33}$. Por otra parte los hijos de madres con anemia ferropriva al momento del parto tienen una mayor prevalencia de anemia ferropriva durante el primer año de vida habiéndose controlado otros factores confundentes ${ }^{38-40}$.

\section{Consecuencias de la deficiencia de hierro sobre el SNC}

Desde el pionero trabajo de Oski ${ }^{41}$ hace 25 años, han aparecido decenas de estudios demostrando inequívocamente que la anemia ferropriva en la infancia produce un retraso del desarrollo psicomotor. Más recientemente, se describió que estos efectos deletéreos persisten hasta los 5 y 10 años. La tercera generación de estas investigaciones ha sido categórica en demostrar alteraciones en la maduración del sistema nervioso central que también persisten más allá de la infancia. Lo más inquietante de estos hallazgos, es que parecieran no ser reversibles, aún a largo plazo, a pesar del tratamiento oportuno y satisfactorio de la anemia de acuerdo con las normas pediátricas habituales. Estos hechos ponen énfasis en la importancia de medidas de prevención de la anemia por carencia de hierro.

Muchas de las investigaciones iniciales adolecieron de los inconvenientes de no contar con adecuados instrumentos de medida de la conducta y dificultades para la identificación de controles no anémicos comparables con los sujetos afectados. Posteriormente, aparecieron trabajos en lactantes de 6 a 24 meses que sortearon adecuadamente estos obstáculos. Entre estos, destacan 2; el estudio de Lozoff et al. ${ }^{42}$ en Costa Rica y el de Walter et al. en Chile ${ }^{43}$. Ambos grupos documentaron que los lactantes con anemia ferropriva presentaban retrasos en el desarrollo psicomotor significativos al compararlos con lactantes suficientes en hierro. También es cierto, que aunque el retraso era leve, éste era consistente. Entre los anémicos, mientras más severa y prolongada era la anemia, más pronunciado el defecto. Curiosamente y coincidiendo con otros estudios, los lactantes con déficit de hierro, pero sin anemia, se comportaron igual a los niños con óptima nutrición en hierro. La explicación de esta aparente paradoja no está clarificada. Sin embargo, en estudios animales sometidos a balance negativo de hierro, se agotan las reservas de hierro tanto en la médula ósea como en el hígado y la mitocondria, mucho antes que descienda la hemoglobina. Es verosímil imaginar, que los niños anémicos deben estar sometidos a una carencia de hierro más severa en los tejidos, necesaria para reflejarse en conducta. También es cierto que los instrumentos para medir desarrollo a esta edad no son muy discriminantes. Como en el estudio chileno todos los niños tenían la misma edad (12 meses), se pudieron examinar las mismas tareas para identificar que áreas específicas eran las más afectadas. En la escala mental: el desarrollo del lenguaje y en la (psico) motora, el equilibrio en la bipedestación y la marcha. 
10 M. OLIVARES \& T. WALTER

Todos los estudios, excepto uno ${ }^{44}$, no han podido demostrar una recuperación de la conducta a corto o mediano plazo (hasta 6 meses). Sin embargo, ya que el desarrollo psicomotor no es un buen predictor del futuro desempeño cognitivo, más importante aún, es necesario estudiar el impacto a más largo plazo en niños mayores. En los 2 estudios descritos anteriormente ${ }^{42,43}$, se siguieron longitudinalmente a los niños hasta los 5 y 10 años. Para evaluar los niños a los 5 años, los investigadores acordaron usar las mismas pruebas y así poder comparar resultados ${ }^{45,46}$. Ambos estudios coincidieron sorprendentemente en todas las funciones; los niños que al año fueron anémicos, tenían un desempeño significativamente inferior a los controles previamente no anémicos. Por ejemplo, en ambos estudios a los 5 años, el Coeficiente Intelectual ( $\mathrm{Cl}$ ) en ambos casos fue 5 puntos inferior en los previamente anémicos. A parte del $\mathrm{Cl}$, las otras pruebas fueron: integración psicomotora; destreza motora fina y gruesa; una escala de preparación para la escolaridad, y una escala de lenguaje expresivo y receptivo. Consistentemente en to das estas pruebas y tanto en Costa Rica como en Chile se hallaron mermas significativas de desempeño en los previamente anémicos en comparación a los controles. Esto adquiere mayor importancia, porque la exploración cognitiva a esta edad es más sofisticada y otorga una buena predicción del desempeño futuro. A los 10-12 años Lozoff et al. ${ }^{47}$ evaluaron a los niños con una batería de pruebas cognitivas, socio-emocionales, motoras y de desempeño escolar. Los niños que habían presentado anemia al año presentaron inferiores resultados en pruebas de función mental y motora. Al controlar estadísticamente por factores de confusión, siguieron exhibiendo desempeño deficiente en aritmética, expresión escrita, motricidad y algunos procesos cognitivos como la memoria espacial, recuerdo selectivo y otros. También fue más frecuente que los anémicos hubieran repetido de curso o haber sido referidos para refuerzo académico. Padres y maestros reportaron problemas conductuales especialmente de ansiedad y depresión, problemas de relación social y déficit atencional.

En resumen, el presentar anemia durante la edad temprana sigue siendo un factor de riesgo para pobre desempeño más de 10 años más tarde. En el seguimiento de los niños chilenos, aunque ninguno de estos niños repitió de curso, se encontró alteraciones motoras finas y al comparar los desempeños escolares, los anémicos tenían notas bajo la mediana de su curso significativamente más frecuentes que los niños controles en aritmética y educación física. Este último hecho es interesante, pues se podría pensar que la educación física sería un ramo de poca discriminación, si embargo era tal la "torpeza" motora de estos niños que fue notoria la diferencia incluso en distintos colegios y evaluados por profesores que no conocían la condición del niño. Reiterando, el presentar anemia durante la edad temprana sigue siendo un factor de riesgo para pobre desempeño más de 10 años después. Estos estudios demuestran que de esa anemia temprana, aún corregida oportunamente los déficit persisten a muy largo plazo.

Sin embargo, las pruebas conductuales a veces son de interpretación incierta, pero en los últimos años se ha determinado alteraciones en parámetros neuro-fisiológicos del sistema nervioso central realizados con métodos más precisos, objetivos y reproducibles. Su relevancia se basa en que el hierro es indispensable para la síntesis y mantención adecuada de la mielina por los oligodendrecitos y este proceso se configura en los primeros dos o tres años de vida, justamente la edad en que es más prevalente la carencia de hierro ${ }^{48}$. Los métodos no invasivos de exploración funcional del SNC que han sido utilizados son los Potenciales Auditivos Evocados del Tronco Cerebral (PAETC) y los Potenciales Evocados Visuales (PEV). La ventaja de estas y otras exploraciones funcionales, es que durante la infancia presentan una progresión predecible y plenamente caracterizada en la maduración que es dependiente de la mielinización de las vías que conducen los impulsos conllevando una 
disminución de la latencia a medida que se completa la mielinización.

Además, mide la conducción nerviosa dentro del SNC y no tan solo de un nervio periférico. En lactantes de 6 meses de edad con anemia ferropriva la latencia o velocidad de la conducción nerviosa central del PAETC, desde la salida de la cóclea al lemnisco lateral del tronco cerebral se encontraba significativamente más lenta que en lactantes con nutrición de hierro adecuada. Al administrarles hierro medicinal por 6 meses, corrigiendo por completo la anemia, persistió esa diferencia al año y a los 18 meses de edad $^{49}$. Más dramático es el hecho que a los 4 años de edad esta prolongación de la conducción nerviosa central persistía e incluso se proyectaba a los 6 años de edad (Figura 1). Esto sin importar cuando se hizo el diagnóstico de la anemia a los 6, 12 o 18 meses de edad. En cuanto al PEV, además de medir la conducción por el nervio óptico, se miden las velocidades de conducción que llegan a la misma corteza occipital. También se encontraron las conducciones más lentas en los lactantes ferroprivos incluso después de la terapia con hierro. Todas estas diferencias son altamente significativas además de ser su magnitud muy marcada, al nivel de - 1 DS o más ${ }^{50}$. Las diferencias funcionales de las vías auditivas y visuales hacen plausible la presencia de alteraciones más generalizadas en mielinización causadas 0 asociadas a la anemia ferropriva. Las anomalías en mielinización podrían explicar las alteraciones vistas en funciones más complejas como el comportamiento y déficits cognitivos, pero por sobre todo deficiencias en la motricidad. Hay abundante literatura conectando mielinización con aprendizaje de habilidades motrices. La disrupción de las vías auditiva y visuales no sólo es importante por si, sino que pueden alterar otras funciones cognitivas interrelacionadas ${ }^{22}$. Hay también evidencia de deficiencias con neurotrasmisores, especialmente dopaminérgicos los cuales están bien identificados como mediadores de circuitos relacionados con la vía auditiva y visual ${ }^{51}$.

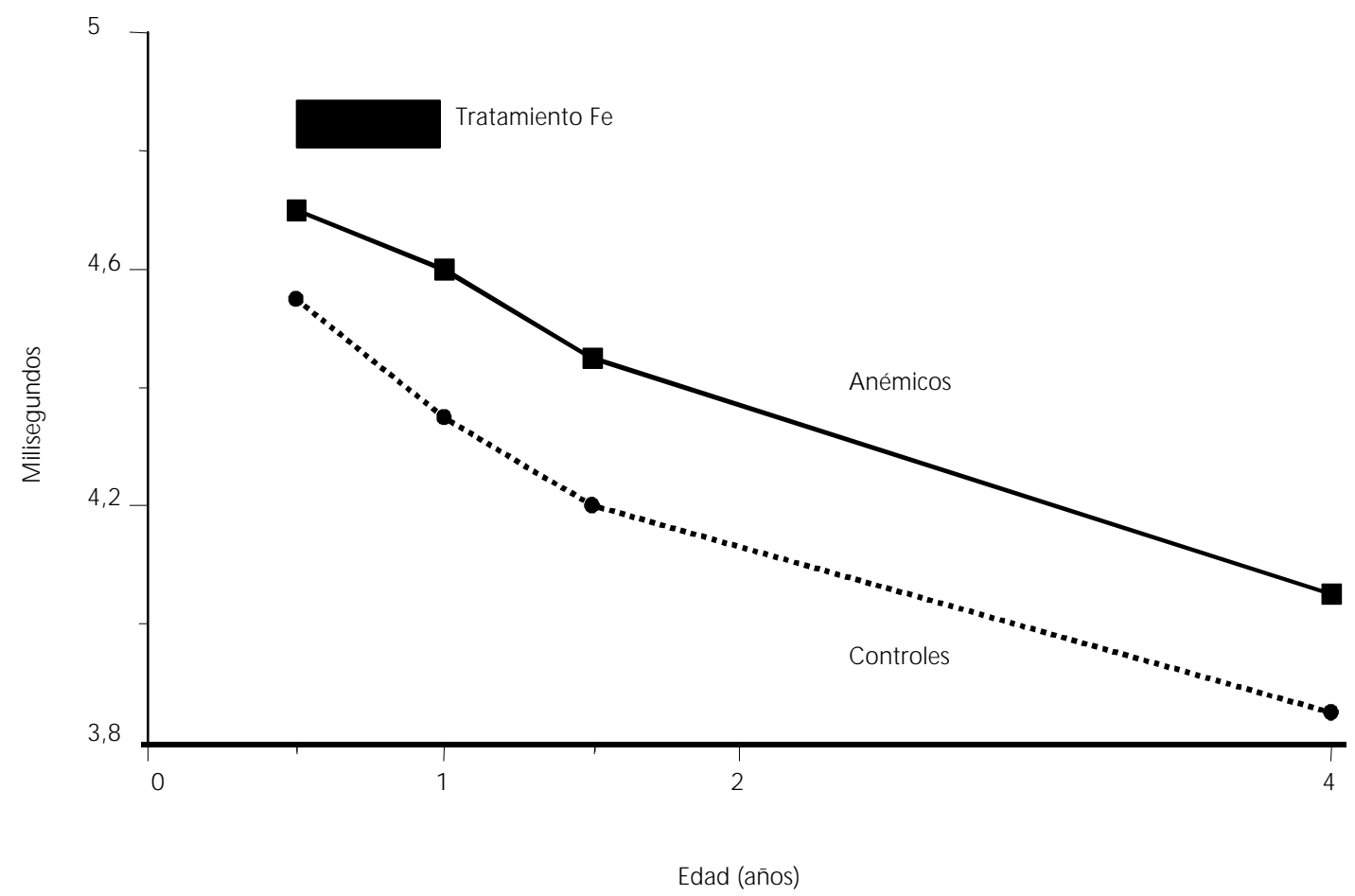

Figura 1. Potencial auditivo de tronco cerebral. Maduración de la conducción central (intervalo I-IV). Se observa que la conducción central del tronco cerebral es mas lenta desde los 6 meses hasta los 4 años de edad, a pesar de un tratamiento controlado durante 6 meses con completa recuperación de la deficiencia de hierro. 
En conclusión, existen pocas dudas que la anemia ferropriva está asociada a algunas consecuencias adversas que parecieran ser definitivamente o difícilmente irreversibles, a pesar de la terapia óptima con hierro. Este hecho enfatiza la prevención de la deficiencia de hierro ya que una vez establecida la anemia tendremos que cargar con las consecuencias antes descritas.

\section{REFERE N C I A S}

1. Bothwell TH, Charlton RW, Cook JD, Finch CA. Iron metabolism in man. Oxford: Blackweel Scientific; 1979.

2. DeMaeyer $E$, Adiels-Tegman $M$. The prevalence of anaemia in the world. World Health Statist Q 1985; 38:302-16.

3. Olivares $M$, Walter $T$, Hertrampf E, Pizarro $F$. Anaemia and iron deficiency disease in children. Br Med Bull 1999; 55:534-48.

4. Olivares M. Anemia ferropriva. En: Meneghello J, Fanta E, Paris E, Puga T, editores. Pediatría. 5. ed. Buenos Aires: Editorial Médica Panamericana; 1997. p.1745-9.

5. Stekel A, Olivares M, Pizarro F, Chadud P, López I, Amar M. Absorption of fortification iron from milk formulas in infants. Am J Clin Nutr 1986; 43:917-22.

6. Saarinen UM, Siimes MA, Dallman PR. Iron absorption in infants: high bioavailability of breast milk as indicated by the extrinsic tag method of iron absorption and by the concentration of serum ferritin. J Pediatr 1977; 91:36-9.

7. Hallberg L, Hultén L. Iron requirements, iron balance and iron deficiency in menstruating and pregnant women. En: Hallberg L, Asp N-G, editores. Iron nutrition in health and disease. London: John Libbey; 1996. p.165-81.

8. International Anemia Consultative Group. Iron deficiency in women. $A$ report of the International Anemia Consultative Group. Washington (DC): Nutrition Foundation; 1981.

9. Hallberg L. Iron balance in pregnancy and lactation. En: Fomon SJ, Zlotkin S, editores. Nutritional anemias. New York: Raven Press; 1992. p.13-28. Nestlé Nutrition Workshop Series; v.30.

10. Food and Agriculture Organization. Requeriments of vitamin A, iron, folate and vitamin B12. Report of a Joint FAO/WHO Expert Consultation. Rome; 1988. p.1-107. Food and Nutrition Series n.23.

11. Hertrampf E, Olivares M, Letelier A, Castillo C. Situación de la nutrición de hierro en la embarazada adolescente al inicio de la gestación. Rev Med Chile 1994; 122:1372-7.

12. Foradori A, Lira P, Grebe $G$, Legues $M E, M$ uñoz B, Vela P. Evaluación de la ferritina sérica en embarazadas y su correlación con otros parámetros hematológicos. Rev Med Chil 1978;106:350-3.

13. Dallman PR. Iron deficiency and related nutritional anemias. En: Nathan DG, Oski FA, editores. Hematology of infancy and childhood. 4th edition. Philadelphia: WB Saunders; 1993. p.413-50.

14. International Anemia Consultative Group. Guidelines for the eradication of iron deficiency anemia. A report of the International Anemia Consultative Group. Washington (DC): Nutrition Foundation. 1977. p.1-29.

15. Olivares $M$, Pizarro $F$, Hertrampf $E$, Walter $T$, Arredondo $M$, Letelier A. Fortificación de Alimentos con hierro en Chile. Rev Chil Nutr 2000; 27:340-4.

16. Allen LH. Anemia and iron deficiency: effects on pregnancy outcome. Am J Clin Nutr 2000; 71:1280S-4S.

17. Walter T, Olivares M, Pizarro F, M uñoz C. Iron, anemia, and infection. Nutr Rev 1997; 55:111-24.

18. Haas JD, Brownlie T IV. Iron deficiency and reduced work capacity: a critical review of the research to determine a causal relationship. J Nutr 2001; 131:676S-90S.

19. Beard JL. Functional consequences of nutritional anemia in adults. En: Ramakrishnan $U$, editor. Nutritional anemias. Boca Raton: CRC Press; 2001. p.111-28.

20. Lozoff B, Wachs TD. Functional correlates of nutritional anemias in infancy and early childhood -child development and behavior. En: 
Ramakrishnan U, editor. Nutritional Anemias. Boca Raton: CRC Press; 2001. p.70-88.

21. Angulo-Kinzler RM, Peirano P, Lozoff B. Spontaneous motor activity in human infants with iron-deficiency anemia. Early Hum Dev 2002; 66:67-79.

22. Peirano P, Algarín C, Garrido M, Roncagliolo M, Lozoff $B$. Interaction of iron deficiency anemia and neurofunctions in cognitive development. En: Fernstrom JD, Uauy R, editores. Nutrition and brain. Basel: Nestec; 2001. p.19-39. Nestlé Nutrition Workshop Series Clinics and Performance Program.

23. Garn SM, Ridella SA, Petzold AS, Falkner F. M aternal hematological levels and pregnancy outcomes. Sem Perinatol 1981;5:155-162.

24. Murphy JF, O'Riordan JO, Newcombe RG, Coles EC, Pearson JF. Relation of haemoglobin levels in first and second trimesters to outcome of pregnancy. Lancet 1986; 1:992-5.

25. Harrison KA, Ibeziako PA. M aternal anaemia and fetal birthweight. J Obstet Gynaecol 1973; 80:798-804.

26. Steer P. Maternal hemoglobin concentration and birth weight. Am J Clin Nutr 2000; 71:1285S-7S.

27. Klebanoff MA, Shiono PH, Selby JV, Trachtenberg Al, Graubard BI. Anemia and spontaneous preterm birth. Am J Obstet Gynecol 1991; 164:59-63.

28. Zhou LM, Yang WW, Hua JZ, Deng CQ, Tao X, Stoltzfus RJ. Relation of hemoglobin measured at different times in pregnancy to preterm birth and low birth weight in Shanghai, China. Am J Epidemiol 1998; 148:998-1006.

29. Hämäläinen $H$, Hakkarainen $K$, Heinonen $S$. Anaemia in the first but not in the second or third trimester is a risk factor for low birth weight. Clin Nutr 2003; 22:271-5.

30. Scholl TO, Hediger ML, Fischer RL, Shearer JW. Anemia vs iron deficiency: increased risk of preterm delivery in a prospective study. Am J Clin Nutr 1992; 55:985-8.

31. Yip R. Significance of an abnormally low or high hemoglobin concentration during pregnancy: special consideration of iron nutrition. Am J Clin Nutr 2000; 72:272S-9S.

32. Lu Lu ZM, Goldenberg RL, Cliver SP, Cutter G, Blankson $\mathrm{M}$. The relationship between maternal hematocrit and pregnancy outcome. Obstet Gynecol 1991; 77:190-4.

33. Ramakrishnan U. Functional consequences of nutritional anemia during pregnancy and early childhood. En: Ramakrishnan U, editor. Nutritional anemias. Boca Raton: CRC Press; 2001. p.44-68.

34. Kelly AM , M cDonald DJ. Observations on maternal and fetal ferritin concentration at term. $\mathrm{Br} J$ Obstet Gynaecol 1978; 85:338-43.

35. M ilman N, Ibsen KK, Christensen JM. Serum ferritin and iron status in mothers and newborn infants. Acta Obstet Gynecol Scand 1987; 66:205-11.

36. Singla PN, Chand S, Khanna S, Agarwal KN. Effect of maternal anaemia on the placenta and the newborn infant. Acta Paediatr Scand 1978; 67:645-8.

37. Mashako L, Preziosi P, Nsibu C, Galán P, Potier de Courcy G, Nsaka T, et al. Iron and folate status in Zairian mothers and their newborns. Ann Nutr Metabol 1991; 35:309-14.

38. de Pee S, Bloem MW, Sari M, Kiess L, Yip R. The high prevalence of low hemoglobin concentration among Indonesian infants aged 3-5 months is related to maternal anemia. J Nutr 2002; 132:2215-21.

39. Colomer J, Colomer C, Gutierrez D, Jubert A, Nolasco A, Donat J, et al. Anaemia during pregnancy as a risk factor for infant iron deficiency: report from the Valencia Infant Anaemia Cohort (VIAC) study. Paediatr Perinat Epidemiol. 1990; 4:196-204.

40. Kilbride J, Baker TG, Parapia LA, Khoury SA, Shuqaidef SW, Jerwood D. Anaemia during pregnancy as a risk factor for iron-deficiency anaemia in infancy: a case-control study in Jordan. Int J Epidemiol 1999; 28:461-8.

41. Oski FA, Honig AS. The effects of therapy on the developmental scores of iron-deficient infants. J Pediatr 1978; 92:21-5. 
14 M. OLIVARES \& T. WALTER

42. Lozoff B, Brittenham GM, Viteri FE, Wolf AW, Urrutia JJ. The effects of short-term oral iron therapy on developmental deficits in iron deficient anemic infants. J Pediatr 1982; 100:351-7.

43. Walter T, de Andraca I, Chadud P, Perales CG. Iron deficiency anemia: adverse effects on infant psychomotor development. Pediatrics 1989; 84:7-17.

44. Idjradinata P, Pollitt E. Reversal of developmental delays in iron-deficient anaemic infants treated with iron. Lancet 1993; 341:1-4.

45. Walter T. Impact of iron deficiency on cognition in infancy and childhood. Eur J Clin Nutr 1993; 47:307-16.

46. Lozoff B, Jimenez E, Wolf AW. Long-Term developmental outcome of infants with iron deficiency. N Engl J Med 1991; 325:687-94.

47. Lozoff B, Jimenez E, Hagen J, Mollen E, Wolf AW. Poorer behavioral and developmental outcome more than 10 years after treatment for iron deficiency in infancy. Pediatrics 2000; 105:E51.

48. Beard J. Iron deficiency alters brain development and functioning. J Nutr 2003; 133:1468S-72S.

49. Roncagliolo M, Garrido M, Walter T, Peirano P, Lozoff $B$. Evidence of altered central nervous system development in infants with iron deficiency anemia at 6 mo: delayed maturation of auditory brainstem responses. Am J Clin Nutr 1998; 68:683-90.

50. Algarin C, Peirano P, Garrido M, Pizarro F, Lozoff B. Iron deficiency anemia in infancy: long-lasting effects on auditory and visual system functioning. Pediatr Res 2003; 53:217-23.

51. Antal A, Keri S, Bodis-Wollner I. Dopamine D2 receptor blockade alters the primary and cognitive components of visual evoked potentials in the monkey, Macaca fascicularis. Neurosci Lett 1997; 232:179-81.

Recebido para publicación en 21 de enero de 2004. 